\title{
LOCALIZATION OF SOLUTIONS OF A NONLINEAR FOKKER-PLANCK EQUATION WITH DIRICHLET BOUNDARY CONDITIONS
}

\author{
B. H. GiLding \\ Faculty of Applied Mathematics, University of Twente, Enschede, The Netherlands \\ (Received 7 September 1988; received for publication 12 October 1988)
}

Key words and phrases: Nonlinear Fokker-Planck equation, nonlinear diffusion-advection, interface, localization, infiltration theory.

\section{INTRODUCTION}

CONSIDER the Cauchy problem for the porous media equation in one space dimension:

$$
\begin{aligned}
u_{t} & =\left(u^{m}\right)_{x x} & & \text { for }(x, t) \in \mathbb{R} \times \mathbb{R}^{+}, \\
u(x, 0) & =u_{0}(x) & & \text { for } x \in \mathbb{R},
\end{aligned}
$$

where $m>1$ and $u_{0}$ is a given nonnegative function. It is well known $[1,11,15]$ that if $u_{0}$ is continuous and bounded, problem (1.1), (1.2) has a unique nonnegative solution defined in a generalized sense, $u(x, t)$. Furthermore, if $u_{0}$ is nontrivial and has compact support one can define the free boundary

$$
\zeta(t)=\sup \{x \in \mathbb{R}: u(x, t)>0\}
$$

for all $t \geq 0$. This interface is continuous and monotonic increasing. Moreover,

$$
\lim _{t \uparrow \infty} \zeta(t)=\infty
$$

Consider though the identical problem for the equations

and

$$
u_{t}=\left(u^{m}\right)_{x x}-\left(u^{n}\right)_{x}
$$

$$
u_{t}=\left(u^{m}\right)_{x x}+\left(u^{n}\right)_{x} .
$$

Under the previously-stated conditions, the Cauchy problem for equation (1.5) admits a unique generalized solution $u(x, t)$ with an interface of the type (1.3) for all $m>1$ and $n \geq 1$. The Cauchy problem for equation (1.6) similarly admits such a solution when $m>\min \{n, 1\}>0[5$, $8,9]$. However, whereas the interface associated with equation (1.5) satisfies (1.4) for all the admissible values of $m$ and $n$, the corresponding interface for equation (1.6) satisfies (1.4) if and only if $n \geq m[4,7,10]$.

The question which we address here is the following. Consider, instead of the abovementioned Cauchy problems, the Cauchy-Dirichlet problem:

$$
\text { (९) } \begin{cases}u_{t}=(a(u))_{x x}+(b(u))_{x} & \text { for }(x, t) \in \mathbb{R}^{+} \times \mathbb{R}^{+}, \\ u(x, 0)=u_{0}(x) & \text { for } x \in \mathbb{R}^{+}, \\ u(0, t)=\psi(t) & \text { for } t \in \mathbb{R}^{+},\end{cases}
$$


with $a \in C([0, \infty)) \cap C^{1}(0, \infty)$ such that $a^{\prime}(s)>0$ for $s>0, b \in C([0, \infty))$, and $u_{0}$ and $\psi$ continuous nonnegative functions on $[0, \infty)$ satisfying the compatibility condition $\psi(0)=u_{0}(0)$. Hypothesize sufficient additional regularity on the functions $a, b$ and $\psi$ to ensure that the problem admits a unique continuous nonnegative generalized solution, $u(x, t)$. Let $u_{0}$ be nontrivial and have compact support, and let $a$ and $b$ be such that the free-boundary

$$
\zeta(t)=\sup (x \in[0, \infty): u(x, t)>0]
$$

is defined for all $t \geq 0$. Then, under what conditions on $a$ and $b$, and in particular on the function $\psi$ is $\sup \{\zeta(t): 0 \leq t<\infty\}<\infty$ ? We shall be especially interested in the answer to this question when the problem takes on the archetypal form

$$
\left(\rho^{*}\right) \begin{cases}u_{r}=\left(u^{m}\right)_{x x}+\lambda\left(u^{n}\right)_{x} & \text { for }(x, t) \in \mathbb{R}^{+} \times \mathbb{R}^{+}, \\ u(x, 0)=u_{0}(x) & \text { for } x \in \mathbb{R}^{+}, \\ u(0, t)=\psi(t) & \text { for } t \in \mathbb{R}^{+},\end{cases}
$$

with $m>1$ when $\lambda=0$, with $m>1$ and $n \geq 1$ when $\lambda<0$, and with $m>\min \{n, 1\}>0$ when $\lambda>0$.

The equation

$$
u_{t}=(a(u))_{x x}+(b(u))_{x}
$$

is often referred to as the nonlinear Fokker-Planck equation $[4,6,8,9]$. When in the above problem $\rho$,

$$
\sup \{\zeta(t): 0 \leq t<\infty\}<\infty,
$$

localization of the solution $u$ is said to occur [6,10-12].

The question posed has some physical relevance. Neglecting the effects of heat and density gradients, sorption and osmosis, the flow of water in a homogeneous isotropic soil is governed by Darcy's law,

$$
\mathbf{q}=-K \operatorname{grad}(h+z)
$$

and conservation of mass,

$$
\frac{\partial \theta}{\partial t}+\operatorname{div} \mathbf{q}=0
$$

Here, $\mathbf{q}$ denotes flux, $K$ hydraulic conductivity, $h$ pressure head, $z$ elevation, $\theta$ moisture content, and $t$ time. Supposing that the flow is essentially one-dimensional in a direction inclined at an angle $\alpha$ to the horizontal, combining (1.9) and (1.10) yields

$$
\frac{\partial \theta}{\partial t}=\frac{\partial}{\partial x}\left\{K \frac{\partial}{\partial x}(h+\lambda x)\right\},
$$

where $x$ indicates distance in the direction of flow and $\lambda=\sin \alpha$. If subsequently hysteresis is negligible and the flow is unsaturated one can rewrite (1.11) as

$$
\frac{\partial \theta}{\partial t}=\frac{\partial}{\partial x}\left\{D(\theta) \frac{\partial \theta}{\partial x}\right\}+\frac{\partial}{\partial x}\{\lambda K(\theta)\},
$$

in which $D(\theta)=K(\theta)(\mathrm{d} h / \mathrm{d} \theta)(\theta)$ denotes the soil-moisture diffusivity [3, 16]. After suitable relabelling this yields equation (1.8) with coefficients $a$ and $b$ which have the properties cited. 
In this derivation of equation (1.8), the coefficient $a$ is the indefinite integral of the soilmoisture diffusivity function, and the coefficient $b$ is the product of the hydraulic conductivity function and the parameter $\lambda$. If the flow is directed downwards then $\lambda<0$, whilst if the flow is horizontal $\lambda=0$, and if the flow is directed upwards $\lambda>0$. Furthermore, the variable $u$ represents unsaturated soil-moisture content. Consequently, if (1.7) defines a free-boundary $\zeta(t)$ this denotes a wetting front separating wet and dry regions of the soil. Seen in this light, the question posed is: what role do soil characteristics, gravity, and boundary conditions play in determining the finite penetration of a wetting front during soil-moisture infiltration?

We are not the first to address this question. For the equation

$$
u_{t}=\left(u^{m}\right)_{x x}+\lambda\left(u^{n}\right)_{x}
$$

with an additional power sink term and boundary data $\psi(t)=A(t+\tau)^{\gamma}$ for some $A>0, \tau>0$ and $\gamma$, the question was earlier posed and studied by Kersner [12]. More recently, for equation (1.12) with more general boundary data, the question has been examined by Diaz and Kersner [6] and by Kersner [13].

Using the results of Kersner [12,13], and, Diaz and Kersner [6], an explicit solution of the porous media equation known as the dipole-type solution [2] and comparison principle arguments; the following conclusions regarding the localization of solutions of problem $P^{*}$ can be drawn.

(i) For $\lambda<0$. Set

$$
\theta_{0}=1, \quad q_{0}(t)=t \quad \text { and } \quad p_{0}(t)=q_{0}(t),
$$

and by induction define

$$
\theta_{k}=\exp \left(\theta_{k-1}\right)
$$

and

$$
q_{k}(t)=\ln \left(q_{k-1}(t)\right) \quad \text { and } \quad p_{k}(t)=q_{k}(t) p_{k-1}(t) \quad \text { for } t \geq \theta_{k}
$$

for all integers $k \geq 1$. If there exists a $k \geq 1$ and a $\delta>0$ such that

$$
\lim _{t \dagger \infty} \inf \left(p_{k}(t) q_{k}^{-\delta}(t)\right)^{1 /(m-1)} \psi(t)>0,
$$

localization does not occur ([13]).

(ii) For $\lambda=0$. Irrespective of the function $\psi$, localization does not occur ([2]).

(iii) For $\lambda>0$, and $n \geq m$. If there exists a $k \geq 1$ and a $\delta>0$ such that (1.16) holds where $p_{k}(t)$ and $q_{k}(t)$ are defined by (1.13)-(1.15), localization does not occur ([13]).

(iv) For $\lambda>0, m>1$ and $n<m$. If there exists a function $\Psi \in C^{\prime}(0, \infty)$ such that $\Psi^{\prime}(t)>0$ for all $t>0, \Psi(t) \uparrow \infty$ as $t \uparrow \infty, \quad \Psi^{\prime}(t)=O\left(\Psi^{1+\varepsilon}(t)\right)$ as $t \uparrow \infty$ for all $\varepsilon>0$, and $\lim _{t+\infty} \inf \psi(t) / \Psi(t)>0$, localization does not occur ([6]).

(v) For $\lambda>0$ and $n<m$. If $\lim _{t \rightarrow \infty} \sup \psi(t)<\infty$, localization occurs [6, 12].

It is evident that the above results do not cover all conceivable permutations of relevant parameters and boundary conditions in problem $\rho^{*}$. In particular, for $\lambda<0$, and for $\lambda>0$ and $n \geq m$, the question of localization when $\psi \equiv 0$ is still left open.

To provide a firm foundation for the present discussion, in the next section we state existence and uniqueness results for problem $\odot$, and review pertinent properties of the interface $\zeta(t)$ 
defined by (1.7). In the section thereafter, we introduce the principal tools with which we shall subsequently tackle the question of localization. These are two comparison principles and an integral identity for solutions of problem $\rho$. We also introduce some useful notation. The remainder of the paper is then devoted to the central question.

Let

$$
S=\left\{v \in(0, \infty): b(s)>b(0) \text { for all } s \in(0, v] \text { and } a^{\prime}(s) /[b(s)-b(0)] \in L^{1}(0, v)\right\} .
$$

In Section 4 we determine sufficient conditions for localization of solutions of problem $\odot$ when the set $S$ is not empty. In Section 5, we correspondingly establish necessary conditions for localization when $S$ is not empty. In Section 6 we study the case of an empty set $S$. We show, under a number of conditions, that in this case localization does not occur.

In the final section, Section 7 , we turn to a particular ramification of the question of localization, namely the question of the possible disappearance of the support of a solution. In [6], Diaz and Kersner showed that if $\lambda>0$ and $n<\min \{m, 1\}$ in problem $\odot^{*}$, and there exists a function $\Psi \in C^{1}(0, \infty)$ such that $\Psi(t)>0>\Psi^{\prime}(t)$ for all $t>0, \Psi(t) \downarrow 0$ as $t \uparrow \infty, \Psi^{\prime}(t)=$ $O\left(\Psi^{1-\varepsilon}(t)\right)$ as $t \uparrow \infty$ for all $\varepsilon>0$, and $\lim _{t+\infty} \sup \psi(t) / \Psi(t)<\infty$, then

$$
\lim _{t \uparrow \infty} \sup \zeta(t)=0 \text {. }
$$

On the other hand, if $\lim \inf \psi(t)>0$, then (1.18) is not the case. We shall extend the results of Diaz and Kersner to the general problem $\mathcal{P}$. Given that localization occurs, we establish conditions under which (1.18) does and does not hold.

Applied to problem $\varphi^{*}$, our results yield the following picture.

(i) For $\lambda \leq 0$. Irrespective of the function $\psi$, localization does not occur.

(ii) For $\lambda>0$ and $n \geq m$. Irrespective of the function $\psi$, localization does not occur.

(iii) For $\lambda>0$ and $n<m$. If $\lim _{i \uparrow \infty} \inf \left\{\psi(t): \tau_{i} \leq t \leq \tau_{i}+\Delta_{i}\right\}=\infty$ for a sequence of intervals $\left\{\left[\tau_{i}, \tau_{i}+\Delta_{i}\right]\right\}_{i=1}^{\infty} \subseteq[0, \infty)$ such that $\Delta_{i} \uparrow \infty$ as $i \uparrow \infty$, localization does not occur.

(iv) For $\lambda>0$ and $n<m$. If $\lim _{t+\infty} \sup \psi(t)<\infty$, localization occurs.

Moreover, for $\lambda>0$ and $n<m$ in problem $\odot^{*}$; if $n \leq 1$ and $\lim _{t+\infty} \sup \psi(t)=0$ then (1.18) holds; whilst if $n>1$ or if $\lim _{i \uparrow \infty} \inf \left\{\psi(t): \tau_{i} \leq t \leq \tau_{i}+\Delta_{i}\right\}>0$ for a sequence of intervals $\left\{\left[\tau_{i}, \tau_{i}+\Delta_{i}\right]\right\}_{i=1}^{\infty} \subseteq[0, \infty)$ such that $\tau_{i} \uparrow \infty$ and $\Delta_{i} \rightarrow 0$ as $i \rightarrow \infty$, then (1.18) is not the case.

In summary, for problem $\rho^{*}$ the only questions left unsettled are those of localization when $\lambda>0$ and $n<m$ and the boundary data function oscillates wildly with increasingly sharper peaks such that

$$
\lim _{t \uparrow \infty} \inf \psi(t)<\infty=\lim _{t \uparrow \infty} \sup \psi(t)
$$

and of the validity of (1.18) when $\lambda>0, n \leq 1$ and the boundary data function again oscillates wildly with increasingly sharper peaks in such a way that

$$
\lim _{t \uparrow \infty} \inf \psi(t)=0<\lim _{t \rightarrow \infty} \sup \psi(t) .
$$

With regard to the physical background to the question of localization, we remark that for soil-moisture infiltration in the direction of gravitational pull or in a horizontal direction, the 
set $S$ defined by (1.17) is automatically empty. On the other hand, for soil-moisture infiltration against the direction of gravitational pull, the emptiness of $S$ is equivalent to the physical characteristic that soil-moisture pressure head becomes unbounded as soil-moisture content decreases to zero; and experimental evidence indicates that this is the case $[3,16]$. Consequently, the question of the finite penetration of a wetting front occurring in soil-moisture infiltration should be interpreted in terms of our results in Section 6.

For soil-moisture infiltration in a direction against the pull of gravity, we indicate a number of conditions which implicate that wetting will carry on in time without bound. In particular, we show that for certain soils, irrespective of the boundary conditions imposed, the penetration of moisture cannot be limited. Thus, in these soils, capillary suction is a sufficiently strong mechanism with regard to upward moisture penetration to override the influence of gravity and any external control of moisture content.

Finally, for soil-moisture flow in the direction of gravitational pull or in a horizontal direction, we show, irrespective of the type of soil and the boundary conditions imposed, that the penetration of soil-moisture cannot be limited. Thus, under circumstances in which soilmoisture infiltration can be viewed as a one-dimensional phenomenon, once moisture is introduced in a soil, whatever its characteristics, lower-lying regions or regions at the same elevation will always eventually become wetted.

\section{PRELIMINARIES}

Equation (1.8) is not assumed to be uniformly parabolic. Hence, problem $P$ need not admit a classical solution [1, 4-12, 15]. In defining a generalized solution to this problem, we follow [5].

Let $D$ denote a domain of the form

$$
D=(0, \infty) \times(\tau, T]
$$

where

$$
0 \leq \tau<T<\infty .
$$

A function $u(x, t)$ is said to be a generalized supersolution of equation (1.7) in $D$ if it is defined, nonnegative, continuous and bounded in $\bar{D}$ and satisfies

$$
\begin{aligned}
& \int_{t_{1}}^{t_{2}} \int_{x_{1}}^{x_{2}}\left\{a(u) \phi_{x x}-b(u) \phi_{x}+u \phi_{t}\right\} \mathrm{d} x \mathrm{~d} t \\
& \quad \leq \int_{x_{1}}^{x_{2}}\left\{u\left(x, t_{2}\right) \phi\left(x, t_{2}\right)-u\left(x, t_{1}\right) \phi\left(x, t_{1}\right)\right\} \mathrm{d} x \\
& \quad+\int_{t_{1}}^{t_{2}}\left\{a\left(u\left(x_{2}, t\right)\right) \phi_{x}\left(x_{2}, t\right)-a\left(u\left(x_{1}, t\right)\right) \phi_{x}\left(x_{1}, t\right)\right\} \mathrm{d} t
\end{aligned}
$$

for all nonempty bounded rectangles $R=\left(x_{1}, x_{2}\right) \times\left(t_{1}, t_{2}\right] \subseteq D$ and nonnegative functions $\phi \in C^{2,1}(\bar{R})$ such that $\phi\left(x_{1}, t\right)=\phi\left(x_{2}, t\right)=0$ for all $t \in\left[t_{1}, t_{2}\right]$. A function $u(x, t)$ is likewise said to be a generalized subsolution of equation (1.8) in $D$ if it satisfies the previous conditions with the inequality sign in (2.3) reversed. A function is a generalized solution of equation (1.8) in $D$ if it is both a generalized supersolution and a subsolution in that domain. Finally, a function $u(x, t)$ is said to be a generalized solution of problem $\theta$ if it is a generalized solution 
of equation (1.8) in all domains of the form (2.1), (2.2) and if it satisfies the imposed initial and boundary-value conditions.

With this definition of a generalized solution of problem $\rho$ existence and uniqueness have been established in [9] under the following hypothesis.

Hypothesis 1. The functions $a, b \in C([0, \infty)) \cap C^{2}(0, \infty)$, and are such that $a^{\prime \prime}$ and $b^{\prime \prime}$ are locally Hölder continuous on $(0, \infty)$, and

$$
a^{\prime}(s)>0 \quad \text { for } s>0 .
$$

The function $u_{0}$ is nonnegative, bounded and continuous on $[0, \infty)$ and the function $\psi$ is nonnegative and continuous on $[0, \infty)$ and satisfies the compatibility condition $\psi(0)=u_{0}(0)$. Furthermore, $s a^{\prime \prime}(s), s a^{\prime}(s) b^{\prime}(s) \in L^{1}(0, \delta)$ for some $\delta>0$, and/or, $a(\psi)$ is locally Lipschitz continuous on $[0, \infty)$.

Under hypothesis 1 the existence of a unique generalized solution $u(x, t)$ of problem $\odot$ was established in [9] by constructing such a solution as the decreasing limit of a sequence of positive classical solutions of equation (1.8). Simultaneously, it was shown that $(a(u))_{x}$ exists and is bounded in the sense of distributions in any set of the form $(\eta, \infty) \times(\tau, \infty)$ with $\eta>0$ and $\tau>0$. Furthermore, setting

$$
Q=(0, \infty) \times(0, \infty)
$$

and

$$
P=\{(x, t) \in Q: u(x, t)>0\},
$$

it was shown that $u \in C^{2,1}(P),(a(u))_{x} \in C^{2,1}(P)$, and $u$ is a classical solution of equation (1.8) in $P$. For problem $\rho^{*}$, hypothesis 1 is satisfied for any $m>0$ and $n>0$, and nonnegative $u_{0} \in C([0, \infty)) \cap L^{\infty}(0, \infty)$ and nonnegative $\psi \in C([0, \infty))$ with $\psi(0)=u_{0}(0)$ irrespective of the Lipschitz continuity of $\psi$.

The next hypothesis is introduced merely for convenience. It involves no loss of generality.

Hypothesis 2. There holds $a(0)=0$ and $b(0)=0$.

Supplementary to hypotheses 1 and 2, we require the following for a precise statement of the central question.

Hypothesis 3. The initial data function $u_{0}$ is nontrivial (i.e. not identically zero) and has compact support.

Hypothesis 4. There are real numbers $\sigma$ and $\delta>0$ such that $\sigma s+b(s)>0$ for all $s \in(0, \delta]$ and $a^{\prime}(s) /\{\sigma s+b(s)\} \in L^{1}(0, \delta)$.

Let $u(x, t)$ denote the unique generalized solution of problem $\odot$ with an initial data function which satisfies hypothesis 3. Utilizing arguments in [8], it can be shown that if hypothesis 4 holds, then $u(x, t)$ has compact support as a function of $x$ for all $t \geq 0$. Hence $\zeta(t)$ is well defined by (1.7). Furthermore, $\zeta(t)$ is lower semi-continuous and continuous from the right on $[0, \infty)$ and uniformly bounded on compact subsets of $[0, \infty)$. On the other hand, utilizing further 
arguments in [8], it can be shown that if hypothesis 4 does not hold then for any $t>0$ either $u(x, t) \equiv 0$ or $\sup \{x \in[0, \infty): u(x, t)>0\}=\infty$. For equation (1.12), hypothesis 4 means that $m>1$ when $\lambda=0, m>1$ and $n \geq 1$ when $\lambda<0$, and $m>\min \{n, 1\}>0$ when $\lambda>0$.

Henceforth, it will automatically be assumed that hypotheses $1-4$ are in force. We use $u(x, t)$ to denote the unique generalized solution of problem $P, \zeta(t)$ to denote the interface subsequently defined by (1.7), and $Q$ and $P$ to denote the sets defined by (2.5) and (2.6) respectively.

\section{THE TOOLS}

In [9], under hypothesis 1 , the generalized solution $u(x, t)$ of problem $\odot$ was constructed as the decreasing limit of a sequence of positive classical solutions of equation (1.8). This construction enables one to deduce the following comparison principles.

LEMma 1. Let $D$ denote a domain of the form (2.1), (2.2).

(i) Let $U(x, t)$ denote a generalized subsolution of equation (1.8) in $D$. Then if $u(x, t) \geq$ $U(x, t)$ for all $(x, t) \in \bar{D} \backslash D$ there holds $u(x, t) \geq U(x, t)$ for all $(x, t) \in \bar{D}$.

(ii) Let $U(x, t)$ denote a generalized supersolution of equation (1.8) in $D$ such that the classical derivative $(a(U))_{x}$ exists and is continuous in a neighbourhood of any point $(x, t) \in D$ where $U(x, t)=0$ and $\{t \in[\tau, T]: U(x, t)>0\}$ is connected for all $x \in[0, \infty)$. Then if $u(x, t) \leq$ $U(x, t)$ for all $(x, t) \in \bar{D} \backslash D$ there holds $u(x, t) \leq U(x, t)$ for all $(x, t) \in \bar{D}$.

Lemma 2. Let $\Omega$ denote a domain of the form $\Omega=\left\{(x, t): \xi_{1}(t)<x<\xi_{2}(t): \tau<t \leq T\right\}$ where $0 \leq \tau<T<\infty$ and $\xi_{1}$ and $\xi_{2}$ are $C([\tau, T])$ functions such that $\xi_{1}(t)<\xi_{2}(t)$ for all $t \in(\tau, T]$. Let $U \in C(\bar{\Omega})$ denote a positive classical subsolution of equation (1.8) in $\Omega$. Then if $u(x, t) \geq U(x, t)$ for all $(x, t) \in \Omega \backslash \Omega$ there holds $u(x, t) \geq U(x, t)$ for all $(x, t) \in \Omega$.

Lemmas 1 and 2 constitute major tools in the ensuing analysis. The third principal tool which we use is an integral identity for solutions of problem $P$. In the context of the description of soil-moisture infiltration by equation (1.8), this identity may be interpreted as the principle of conservation of momentum.

LEMMA 3. For any $T>0$,

$$
\int_{0}^{\infty} x u(x, T) \mathrm{d} x=\int_{0}^{\infty} x u_{0}(x) \mathrm{d} x+\int_{0}^{T} a(\psi(t)) \mathrm{d} t-\int_{0}^{T} \int_{0}^{\infty} b(u(x, t)) \mathrm{d} x \mathrm{~d} t
$$

Proof. Since $\zeta(t)$ is uniformly bounded on compact subsets of $[0, \infty)$, we may choose an $X \in(0, \infty)$ such that $\zeta(t)<X$ for all $t \in[0, T]$. Set

$$
R=\left(x_{1}, x_{2}\right) \times\left(t_{1}, t_{2}\right]=(0,2 X) \times(0, T]
$$

and let $\phi \in C^{2,1}(\bar{R})$ be nonnegative and such that

$$
\phi(x, t)=x \quad \text { for all }(x, t) \in[0, X] \times[0, T]
$$

and

$$
\phi(2 X, t)=0 \quad \text { for all } t \in[0, T]
$$


Then substituting $R$ and $\phi$ in inequality (2.3) of the definition of $u$ as a generalized supersolution and a generalized subsolution of equation $(1.8)$ in $(0, \infty) \times(0, T],(3.1)$ is immediate.

We close this section by introducing some notation which will be continually referred to in the remainder of the paper.

For $v \in(0, \infty)$, we let

$$
\sigma_{\nu}=\sup \{-b(s) / s: 0<s \leq v\}
$$

and

$$
\sigma_{0}=\limsup _{s \downarrow 0}\{-b(s) / s\}=\lim _{\nu \downarrow 0} \sigma_{\nu}
$$

Remark 1 [8]. If hypothesis 4 holds, then $-\infty<\sigma_{\nu}<\infty$ for all $v \in(0, \infty)$. Moreover, given any $v \in(0, \infty)$ and $\sigma>\sigma_{\nu}$ :

$$
\sigma s+b(s)>0 \quad \text { for all } s \in(0, v]
$$

and

$$
a^{\prime}(s) /\{\sigma s+b(s)\} \in L^{1}(0, v) .
$$

For $v \in(0, \infty)$ and $\sigma>\sigma_{\nu}$ we set

$$
I(\sigma, v)=\int_{0}^{\nu} a^{\prime}(s) /\{\sigma s+b(s)\} \mathrm{d} s .
$$

Furthermore, we define the set $S$ by

$$
S=\left\{v \in(0, \infty): b(s)>0 \text { for all } s \in(0, v] \text { and } a^{\prime}(s) / b(s) \in L^{1}(0, v)\right\}
$$

and for nonempty $S$ define

$$
\mu=\sup S \text {. }
$$

Using the definition of $S$ and remark 1 one has the following.

Remark 2. If the set $S$ is not empty then $\sigma_{0} \leq \sigma_{\nu} \leq 0$ for all $\nu \in(0, \mu)$. Whilst if the set $S$ is empty then $\sigma_{v} \geq \sigma_{0} \geq 0$ for all $\nu \in(0, \infty)$.

Finally, for any real variable $y$, we set

$$
[y]_{+}=\max \{0, y\} \text {. }
$$

\section{SUFFICIENT CONDITIONS WHEN $S$ IS NOT EMPTY}

In this section we establish a number of conditions which are sufficient for localization of solutions of problem $\odot$ when the set $S$ defined by (3.3) is not empty. The key to these conditions is the next lemma. We refer to the previous sections for an explanation of the notation used.

LEMMA 4. Let $D$ be a domain of the form (2.1), (2.2), and

$$
v=\sup \{u(x, t):(x, t) \in \widetilde{D} \backslash D\}
$$


Then given any $\sigma>\sigma_{\nu}$,

$$
\zeta(T) \leq[\zeta(\tau)+\sigma(T-\tau)]_{+}+I(\sigma, v) .
$$

Moreover, if

$$
\psi(t)=0 \quad \text { for all } t \in[\tau, T],
$$

then

$$
\zeta(T) \leq[\zeta(\tau)+\sigma(T-\tau)+I(\sigma, \nu)]_{+} .
$$

Proof. We use a comparison argument with a suitably constructed "travelling-wave solution" of equation (1.8). Let

$$
\eta=\max \{\zeta(\tau),-\sigma(T-\tau)\} \quad \text { and } \quad \rho=\eta+I(\sigma, \nu),
$$

and define the function $z(x, t)$ by

$$
\begin{gathered}
z(x, t)=v \quad \text { for } x \leq \eta+\sigma(t-\tau) \\
\int_{z(x, t)}^{\nu} a^{\prime}(s) /\{\sigma s+b(s)\} \mathrm{d} s=x-\eta-\sigma(t-\tau) \quad \text { for } \eta+\sigma(t-\tau)<x<\rho+\sigma(t-\tau) \\
z(x, t)=0 \quad \text { for } x \geq \rho+\sigma(t-\tau) .
\end{gathered}
$$

It is easily verified that $z(x, t)$ is a generalized supersolution of equation (1.8) in $D$ which satisfies the conditions for application of lemma 1. Moreover,

$$
\begin{array}{ll}
u(x, \tau) \leq \nu=z(x, \tau) & \text { for all } x \in[0, \zeta(\tau)), \\
u(x, \tau)=0 \leq z(x, \tau) & \text { for all } x \in[\zeta(\tau), \infty),
\end{array}
$$

and

$$
u(0, t) \leq v=z(0, t) \quad \text { for all } t \in[\tau, T]
$$

Hence, by lemma 1 ,

$$
u(x, t) \leq z(x, t) \quad \text { for all }(x, t) \in \bar{D} .
$$

However, this means that

$$
\zeta(t) \leq \rho+\sigma(t-\tau)
$$

for all $t \in[\tau, T]$, and thus in particular that (4.1) holds.

If (4.2) holds, to derive the second conclusion of the lemma, the above argument may be repeated with

$$
\eta=\max \{\zeta(\tau),-\sigma(T-\tau)-I(\sigma, v)\}
$$

LEMma 5. Suppose that $S$ is not empty, and there is a $v, 0<v \leq \mu$, and a $\tau, 0 \leq \tau<\infty$, such that

and

$$
\begin{aligned}
\psi(t) \leq v & \text { for all } t \in[\tau, \infty) \\
u(x, \tau) \leq v & \text { for all } x \in[0, \infty)
\end{aligned}
$$

Then, localization occurs.

$$
a^{\prime}(s) / b(s) \in L^{1}(0, v)
$$


Proof. For $T \in(\tau, \infty)$ let

$$
M=\max \{\sup \{\psi(t): \tau \leq t \leq T\}, \sup \{u(x, \tau): 0 \leq x<\infty\}\} .
$$

Note that

By lemma 4,

$$
0<M<\infty \quad \text { and } \quad M \leq v
$$

$$
\zeta(T) \leq[\zeta(\tau)+\sigma(T-t)]_{+}+I(\sigma, M)
$$

for all $\sigma>\sigma_{M}$. However, since by remark $2, \sigma_{M} \leq 0$, and by hypothesis $a^{\prime}(s) / b(s) \in L^{1}(0, M)$, we may let $\sigma \downarrow 0$ in (4.5) to deduce

$$
\zeta(T) \leq \zeta(\tau)+\int_{0}^{M} a^{\prime}(s) / b(s) \mathrm{d} s .
$$

Whence

$$
\zeta(T) \leq \zeta(\tau)+\int_{0}^{v} a^{\prime}(s) / b(s) \mathrm{d} s
$$

for all $T \in[\tau, \infty)$.

Our first theorem follows immediately from lemma 5 .

THEOREM 1. Suppose that $\mu=\infty$ and $a^{\prime}(s) / b(s) \in L^{1}(0, \infty)$. Then, irrespective of the function $\psi$, localization occurs.

When the conditions of theorem 1 do not hold, we need an estimate of the supremum of $u(x, t)$ in terms of the supremum of $\psi$ to fully benefit from lemma 5 . The next lemma fits the bill.

Lemma 6. Suppose that $S$ is not empty and $\psi^{*}=\lim _{t \dagger \infty} \sup \psi(t)<\mu$. Then given any $\nu \in\left(\psi^{*}, \mu\right)$ there is a $\tau \in[0, \infty)$ such that (4.3) and (4.4) hold.

Proof. By hypothesis, given any $v \in\left(\psi^{*}, \mu\right)$ we can find a $\tau_{0} \in[0, \infty)$ such that $\psi(t) \leq v$ for all $t \in\left[\tau_{0}, \infty\right)$. To prove the lemma, it therefore suffices to show that there is a $\tau \in\left[\tau_{0}, \infty\right)$ such that (4.4) holds. In view of lemma 1 , without loss of generality we may suppose that $\psi(t) \equiv v$ for all $t \in\left[\tau_{0}, \infty\right)$ and that $u\left(x, \tau_{0}\right)$ has at most one maximum on $[0, \infty)$. Since $u$ is a classical solution of equation (1.8) in $P$, by a lap-number argument [14], it then follows that $u(x, t)$ has at most one maximum on $[0, \infty)$ for any $t \geq \tau_{0}$. Moreover, setting

$$
M(t)=\sup \{u(x, t): x \in[0, \infty)\},
$$

$M(t)$ is a decreasing function on $\left[\tau_{0}, \infty\right)$.

Suppose now that $M(T)>v$ for some $T \in\left(\tau_{0}, \infty\right)$. Because $u$ is continuous in $Q, u(x, t) \equiv v$ for all $(x, t) \in[0,0] \times\left[\tau_{0}, T\right], 0<v<M(T)$, and $[0,0] \times\left[\tau_{0}, T\right]$ is compact; there is an $\eta \in(0, \infty)$ such that

$$
0<u(x, t)<M(T) \quad \text { for all }(x, t) \in[0, \eta] \times\left[\tau_{0}, T\right] .
$$


Since though, $u(x, t)$ has at most one maximum on $[0, \infty)$ and $u(0, t)=v<M(T) \leq M(t)$ for all $t \in\left[t_{0}, T\right]$, this can only be the case if

$$
v \leq u(x, t) \quad \text { for all }(x, t) \in[0, \eta] \times\left[\tau_{0}, T\right] .
$$

Pick $\delta \in(0, \eta)$. By lemma 3,

$$
\int_{0}^{\infty} x u(x, T) \mathrm{d} x+\int_{\tau_{0}}^{T} \int_{0}^{\infty} b(u(x, t)) \mathrm{d} x \mathrm{~d} t=\int_{0}^{\infty} x u\left(x, \tau_{0}\right) \mathrm{d} x+\int_{\tau_{0}}^{\tau} a(\psi(t)) \mathrm{d} t .
$$

Also, by lemma 3 applied to the generalized solution $\hat{u}(x, t)=u(x+\delta, t)$,

$$
\int_{\delta}^{\infty}(x-\delta) u(x, T) \mathrm{d} x+\int_{\tau_{0}}^{T} \int_{\delta}^{\infty} b(u(x, t)) \mathrm{d} x \mathrm{~d} t=\int_{\delta}^{\infty}(x-\delta) u\left(x, \tau_{0}\right) \mathrm{d} x+\int_{\tau_{0}}^{T} a(u(\delta, t)) \mathrm{d} t .
$$

Hence, subtracting (4.8) from (4.7), and recalling that $\psi(t)=v$ for all $t \geq \tau_{0}$,

$$
\begin{aligned}
& \int_{0}^{\delta} x u(x, T) \mathrm{d} x+\delta \int_{\delta}^{\infty} u(x, T) \mathrm{d} x+\int_{\tau_{0}}^{T} \int_{0}^{\delta} b(u(x, t)) \mathrm{d} x \mathrm{~d} t \\
& \quad=\int_{0}^{\delta} x u\left(x, \tau_{0}\right) \mathrm{d} x+\delta \int_{\delta}^{\infty} u\left(x, \tau_{0}\right) \mathrm{d} x+\int_{\tau_{0}}^{T}[a(v)-a(u(\delta, t))] \mathrm{d} t \\
& \quad \leq \int_{0}^{\delta} x u\left(x, \tau_{0}\right) \mathrm{d} x+\delta \int_{\delta}^{\infty} u\left(x, \tau_{0}\right) \mathrm{d} x
\end{aligned}
$$

by (2.4) and (4.6). Subsequently, dividing by $\delta$ and letting $\delta \downarrow 0$,

$$
\int_{0}^{\infty} u(x, T) \mathrm{d} x+\int_{\tau_{0}}^{T} b(\psi(t)) \mathrm{d} t \leq \int_{0}^{\infty} u\left(x, \tau_{0}\right) \mathrm{d} x .
$$

So that

$$
\int_{\tau_{0}}^{T} b(v) \mathrm{d} t<\int_{0}^{\infty} u\left(x, \tau_{0}\right) \mathrm{d} x .
$$

It follows that if $M(T)>v$ then

$$
T<\tau_{0}+\int_{0}^{\infty} u\left(x, \tau_{0}\right) \mathrm{d} x / b(v) .
$$

Looking at this statement in negation, it says that if $T$ is large enough then $M(T) \leq v$. Hence we can indeed choose a $\tau \in\left[\tau_{0}, \infty\right)$ such that (4.4) holds.

Combining lemmas 5 and 6 we obtain the following theorem.

THEOREM 2. Suppose that $S$ is not empty. Then, if $\lim _{t \rightarrow \infty} \sup \psi(t)<\mu$, localization occurs.

Corollary 2.1. Let $\lambda>0$ and $n<m$ in problem $\mathcal{P}^{*}$. Then, if $\lim \sup \psi(t)<\infty$, localization occurs. 
Recalling the derivation of equation (1.8) in the theory of soil-moisture infiltration, theorems 1 and 2 state that should soil-moisture pressure head be bounded for small moisture contents, then localization of wetting fronts in an upward direction may occur.

\section{NECESSARY CONDITIONS WHEN $S$ IS NOT EMPTY}

Whereas the previous section indicated conditions under which localization may occur when the set $S$ is not empty, the purpose of this section is to indicate conditions under which localization is excluded when the set $S$ is not empty. We begin with a counterpart to lemma 4 .

Lemma 7. Let $0 \leq \tau<T<\infty$. Suppose that there is a nonnegative function $\xi_{1} \in C([\tau, T])$ such that

$$
u\left(\xi_{1}(t), t\right) \geq v \quad \text { for all } t \in[\tau, T]
$$

for some $v \in(0, \infty)$. Then, given any $\sigma>\left[\sigma_{\nu}\right]_{+}$,

$$
\zeta(T) \geq \min \{\sigma(T-\tau), I(\sigma, v)\} .
$$

Proof. We again use a comparison principle argument with a suitably constructed "travelling-wave solution" of equation (1.8). Let

$$
\eta=\min \{0, I(\sigma, v)-\sigma(T-\tau)\}
$$

and

$$
\xi_{2}(t)=\eta+\sigma(t-\tau) \quad \text { for all } t \in[\tau, T]
$$

Since, if $\xi_{2}(T) \leq \xi_{1}(T)$ the lemma is trivially true, without loss of generality we may suppose that

$$
\xi_{1}(T)<\xi_{2}(T)
$$

However,

$$
\xi_{2}(\tau) \leq 0 \leq \xi_{1}(\tau)
$$

So there is a $\tau^{*} \in[\tau, T)$ such that

$$
\xi_{1}\left(\tau^{*}\right)=\xi_{2}\left(\tau^{*}\right)
$$

and

$$
\xi_{1}(t)<\xi_{2}(t) \quad \text { for all } t \in\left(t^{*}, T\right] .
$$

Consider the function $z(x, t)$ defined in the closure of the domain

$$
\Omega^{*}=\left\{(x, t): 0<x<\xi_{2}(t) \text { and } \tau<t \leq T\right\}
$$

by

$$
\int_{0}^{z(x, t)} a^{\prime}(s) /\{\sigma s+b(s)\} \mathrm{d} s=\sigma(t-\tau)-x+\eta .
$$

It is easily verified that $z$ is a classical travelling-wave solution of equation $(1.8)$ in $\Omega^{*}$. Moreover,

$$
z(x, t) \leq z(0, T) \leq v \quad \text { for all }(x, t) \in \Omega^{*} .
$$


Hence, defining

$$
\Omega=\left\{(x, t) \in \Omega^{*}: \xi_{1}(t)<x \text { and } \tau^{*}<t\right\},
$$

$z(x, t)$ is a positive classical solution of (1.8) in $\Omega$ for which

$$
z\left(\xi_{1}(t), t\right) \leq v \leq u\left(\xi_{1}(t), t\right) \quad \text { for all } t \in\left[\tau^{*}, T\right]
$$

and

$$
z\left(\xi_{2}(t), t\right)=0 \leq u\left(\xi_{2}(t), t\right) \quad \text { for all } t \in\left[\tau^{*}, T\right] .
$$

Therefore, by the comparison principle lemma 2 ,

$$
z(x, t) \leq u(x, t) \quad \text { for all }(x, t) \in \Omega .
$$

This gives $\zeta(T) \geq \xi_{2}(T)$ or equivalently (5.1).

We now turn to the main result of this section.

THEOREM 3. Suppose that $S$ is not empty and $a^{\prime}(s) / b(s) \notin L^{1}(0, \mu)$. Then, if

$$
\lim _{i \mid \infty} \inf \left\{\psi(t): \tau_{i} \leq t \leq \tau_{i}+\Delta_{i}\right\} \geq \mu
$$

for a sequence of intervals $\left\{\left[\tau_{i}, \tau_{i}+\Delta_{i}\right]\right\}_{i=1}^{\infty} \subseteq[0, \infty)$ such that $\Delta_{i} \uparrow \infty$ as $i \uparrow \infty$, localization does not occur.

Proof. Suppose to the contrary of the statement of the theorem, that there is an $X \in(0, \infty)$ such that

$$
\zeta(t)<X \quad \text { for all } t \in[0, \infty) .
$$

Then, since $a^{\prime}(s) / b(s) \notin L^{1}(0, \mu)$, we can choose a $v \in S$ such that

$$
X<\int_{0}^{v} a^{\prime}(s) / b(s) \mathrm{d} s<\infty .
$$

Subsequently, we can also find a $\sigma>0$ such that

$$
X<\int_{0}^{\nu} a^{\prime}(s) /\{\sigma s+b(s)\} \mathrm{d} s=I(\sigma, v)<\infty .
$$

We let $i$ be so large that

$$
\psi(t) \geq v \quad \text { for all } t \in\left[\tau_{i}, \tau_{i}+\Delta_{i}\right]
$$

and

$$
\sigma \Delta_{i}>X
$$

Now, by lemma 7 with $\xi_{1} \equiv 0$ on $\left[\tau_{i}, \tau_{i}+\Delta_{i}\right]$,

$$
\zeta\left(\tau_{i}+\Delta_{i}\right) \geq \min \left\{\sigma \Delta_{i}, I(\sigma, v)\right\}>X,
$$

which contradicts (5.2). Thus (5.2) cannot be true. 
Corollary 3.1. Let $\lambda>0$ and $n<m$ in problem $\rho^{*}$. Then, if

$$
\lim _{i \mid \infty} \inf \left\{\psi(t): \tau_{i} \leq t \leq \tau_{i}+\Delta_{i}\right\}=\infty
$$

for a sequence of intervals $\left\{\left[\tau_{i}, \tau_{i}+\Delta_{i}\right]\right\}_{i=1}^{\infty} \subseteq[0, \infty)$ such that $\Delta_{i} \uparrow \infty$ as $i \uparrow \infty$, localization does not occur.

Theorem 3 can be viewed as the complement of theorems 1 and 2 . For suppose that

$$
\begin{aligned}
\lim _{t \uparrow \infty} \inf \psi(t) & =\lim _{t \uparrow \infty} \sup \psi(t) \\
& =\psi^{*} \text { say. }
\end{aligned}
$$

Let $\mu=\infty$. Then theorems 1 and 2 state that localization occurs if $a^{\prime}(s) / b(s) \in L^{1}(0, \infty)$ or $\psi^{*}<\infty$. Whilst theorem 3 states that localization occurs only if $a^{\prime}(s) / b(s) \in L^{1}(0, \infty)$ or $\psi^{*}<\infty$. Correspondingly, when $\mu<\infty$, theorem 2 implies that localization occurs if $\psi^{*}<\mu$, whilst theorem 3 implies that localization occurs only if $\psi^{*}<\mu$.

Note though that for the absence of localization theorem 3 does not require the restrictive condition $\lim _{t \uparrow \infty} \inf \psi(t) \geq \mu$. To be able to apply the theorem it is merely sufficient that there is an unbounded sequence of intervals on which the infimum of $\psi$ is greater than or equal to $\mu$. Thus it is possible that $\lim \inf \psi(t)<\mu$ while localization is still excluded.

\section{THE SET $S$ IS EMPTY}

This section is devoted to the question of localization when the set $S$ defined by (3.3) is empty. We shall establish a number of conditions which preclude localization.

We use the following lemmas.

Lemma 8. Let $0 \leq \tau<T<\infty$. Suppose that there is a nonnegative function $\xi \in C([\tau, T])$ such that

$$
u(\xi(t), t)>0 \quad \text { for all } t \in[\tau, T]
$$

Then

$$
\zeta(T)>\xi(\tau)+\sigma_{0}(T-\tau)
$$

Proof. The lemma may be proved by adapting arguments in [8]. We omit the details.

LEMMA 9. Suppose that $\sigma_{0} \geq 0$ and there are real numbers $\rho$ and $\delta>0$ such that

$$
p s-b(s)>0 \quad \text { for all } s \in(0, \delta]
$$

and

$$
a^{\prime}(s) /\{\rho s-b(s)\} \in L^{1}(0, \delta)
$$

Then

$$
\zeta(t) \geq \zeta(0)+\sigma_{0} t \quad \text { for all } t \geq 0
$$


Proof. Without loss of generality we may suppose that $\rho>0$. Set

Let

$$
V=\left\{t \in[0, \infty): \zeta(t) \geq \zeta(0)+\sigma_{0} t\right\} .
$$

and let $\varepsilon^{*} \in(0, \delta)$ be so small that

$$
\Delta=\frac{1}{2} \zeta(0) / p>0
$$

$$
\left.\rho \Delta<\zeta(0)-\varepsilon-\int_{0}^{\varepsilon} a^{\prime}(s) / \rho s-b(s)\right\} \mathrm{d} s
$$

for all $\varepsilon \in\left(0, \varepsilon^{*}\right)$.

Suppose now that $\tau \in V$. Set $T=\tau+\Delta$ and $D=(0, \infty) \times(\tau, T]$. For $\varepsilon \in\left(0, \varepsilon^{*}\right)$ let $u_{0, \varepsilon}(x) \in C(-\infty, \infty)$ be such that

$$
\begin{aligned}
0 & \leq u_{0, \varepsilon}(x) \leq \varepsilon & & \text { for all } x \in(-\infty, \infty), \\
u_{0, \varepsilon}(x) & \leq u(x, \tau) & & \text { for all } x \geq 0,
\end{aligned}
$$

and the sets of points $\left\{x \in(-\infty, \infty): u_{0, \varepsilon}(x)>0\right\}$ is a nonempty connected subinterval of $(\zeta(\tau)-\varepsilon, \zeta(\tau))$. Next, let $u_{\varepsilon}(x, t)$ denote the generalized solution of the Cauchy problem for equation $(1.8)$ in $(-\infty, \infty) \times(\tau, T]$ with initial data $u_{\varepsilon}(x, \tau)=u_{0, \varepsilon}(x)$ for all $x \in(-\infty, \infty)$ whose existence was established in [9]. By results in [8],

$$
u_{\varepsilon}(x, t)=0 \quad \text { for all } x \leq \zeta(\tau)-\varepsilon-\rho(t-\tau)-\int_{0}^{\varepsilon} a^{\prime}(s) /\{\rho s-b(s)\} \mathrm{d} s
$$

and $t \in[\tau, T]$. Whence

$$
u_{\varepsilon}(x, t)=0 \quad \text { for all }(x, t) \in(-\infty, 0] \times[\tau, T] .
$$

Consequently it can be shown that $u_{\varepsilon}(x, t)$ is a generalized solution of equation (1.8) in $D$ for which $u_{\varepsilon}(0, t)=0 \leq u(0, t)$ for all $t \in[\tau, T]$ and (6.3) holds. So, invoking lemma 1 ,

$$
u_{\varepsilon}(x, t) \leq u(x, t) \quad \text { for all }(x, t) \in \bar{D} .
$$

However, by a further argument in [8], there exists a $\xi \in C([\tau, T])$ such that

$$
u_{\varepsilon}(\xi(t), t)>0 \quad \text { for all } t \in[\tau, T]
$$

and by (6.4) this function $\xi$ must be nonnegative. Combining (6.5) with (6.6) there is a nonnegative function $\xi \in C([\tau, T])$ such that

$$
u(\xi(t), t)>0 \quad \text { for all } t \in[\tau, T] .
$$

Consequently, lemma 8 informs us that

$$
\zeta(t) \geq \zeta(\tau)-\varepsilon+\sigma_{0}(t-\tau) \quad \text { for all } t \in[\tau, T] .
$$

Letting $\varepsilon \downarrow 0$ yields

$$
\zeta(t) \geq \zeta(\tau)+\sigma_{0}(t-\tau) \quad \text { for all } t \in[\tau, T]
$$

Whence, since $\tau \in V$,

$$
\zeta(t) \geq \zeta(0)+\sigma_{0} t \quad \text { for all } t \in[\tau, \tau+\Delta] .
$$

We have consequently shown that if $\tau \in V$ then $[\tau, \tau+\Delta] \subseteq V$. However, since trivially $0 \in V$, this implies that $V=[0, \infty)$. 
We are now in a position to state our first result on the question of localization when the set $S$ is empty.

THEOREM 4. Suppose that $\sigma_{0}>0$ and there are real numbers $\rho$ and $\delta>0$ such that (6.1) and (6.2) hold. Then, irrespective of the function $\psi$, localization does not occur.

The condition that there are constants $\rho$ and $\delta>0$ such that (6.1) and (6.2) hold is equivalent to hypothesis 4 with $b(s)$ replaced by $-b(s)$. Thus this condition is tantamount to saying that the equation displays finite speed of propagation to the left. In terms of soil-moisture infiltration, this means that moisture cannot flow away too quickly. As an example of a pair of functions which satisfy the basic hypotheses given by hypotheses 1-4 but do not conform to the additional hypothesis of theorem 4 , consider

$$
a(s)=s^{m} \quad \text { with } m>1
$$

and

$$
b(s)=-\sigma_{0} s+\beta s^{1 / 2}\left\{1+\sin s^{-1}\right\} \quad \text { with } \beta>0 .
$$

For this case, we shall prove the following weaker result.

Theorem 5. Suppose that $\sigma_{0}>0$. Then, if $\inf \left\{\psi(t): \tau_{i} \leq t \leq \tau_{i}+\Delta_{i}\right\}>0$ for a sequence of intervals $\left\{\left[\tau_{i}, \tau_{i}+\Delta_{i}\right]\right\}_{i=1}^{\infty} \subseteq[0, \infty)$ such that $\Delta_{i} \uparrow \infty$ as $i \uparrow \infty$, localization does not occur.

Proof. By lemma 8 with $\xi \equiv 0$ on $\left[\tau_{i}, \tau_{i}+\Delta_{i}\right]$,

$$
\zeta\left(\tau_{i}+\Delta_{i}\right)>\sigma_{0} \Delta_{i}
$$

for all $i \geq 1$. Whence, letting $i \uparrow \infty, \lim _{i \uparrow \infty} \sup \zeta(t)=\infty$.

Theorems 4 and 5 are both obtained under the constraint that $\sigma_{0}>0$. Our next results also apply when $\sigma_{0}=0$. We prepare their way with two lemmas.

Lemma 10. Suppose that $S$ is empty and $\sigma_{0}=0$. Then given any $v>0$,

$$
I(\sigma, v) \uparrow \infty \quad \text { as } \sigma \downarrow \sigma_{\nu} .
$$

Proof. Suppose that the lemma is untrue. Then, since the integrand in (3.2) is a nonincreasing function of $\sigma$, by the monotone convergence theorem there must hold

$$
a^{\prime}(s) /\left\{\sigma_{\nu} s+b(s)\right\} \in L^{1}(0, v),
$$

which, in view of (2.4) also requires

$$
\sigma_{\nu} s+b(s)>0 \quad \text { for all } s \in(0, v] .
$$

If though $\sigma_{\nu}>\sigma_{0}$, by the definition of these parameters, (6.10) cannot possibly hold. Whilst, on the other hand, if $\sigma_{v}=\sigma_{0}=0$, then (6.9), (6.10) violates the assumption that $S$ is empty. The conclusion must be that the lemma is true. 
Lemma 11. Suppose that $S$ is empty. Suppose furthermore that there is a $v>0$ and a sequence of intervals $\left\{\left[\tau_{i}, \tau_{i}+\Delta_{i}\right]\right\}_{i=1}^{\infty} \subseteq[0, \infty)$ such that $\Delta_{i} \uparrow \infty$ as $i \uparrow \infty$ and for each interval there is a nonnegative function $\xi_{i} \in C\left(\left[\tau_{i}, \tau_{i}+\Delta_{i}\right]\right)$ for which

$$
u\left(\xi_{i}(t), t\right) \geq v \quad \text { for all } t \in\left[\tau_{i}, \tau_{i}+\Delta_{i}\right]
$$

Then, localization does not occur.

Proof. We recall that since the set $S$ is empty, $\sigma_{v} \geq \sigma_{0} \geq 0$. By lemma 7 ,

$$
\zeta\left(\tau_{i}+\Delta_{i}\right) \geq \min \left\{\sigma \Delta_{i}, I(\sigma, v)\right\}
$$

for all $i \geq 1$ and $\sigma>\sigma_{\nu}$. Hence

$$
\lim _{t \uparrow \infty} \sup \zeta(t) \geq I(\sigma, v)
$$

Whence, if $\sigma_{0}=0, \lim _{t \uparrow \infty} \sup \zeta(t)=\infty$ by lemma 10 . On the other hand, if $\sigma_{0}>0$,

$$
\zeta\left(\tau_{i}+\Delta_{i}\right)>\sigma_{0} \Delta_{i}
$$

for all $i \geq 1$ by lemma 8 . So in this case also $\lim _{t \rightarrow \infty} \sup \zeta(t)=\infty$.

From lemma 11 we immediately obtain our next theorem.

Theorem 6. Suppose that $S$ is empty. Then, if $\lim _{i \uparrow \infty} \inf \left\{\psi(t): \tau_{i} \leq t \leq \tau_{i}+\Delta_{i}\right\}>0$ for a sequence of intervals $\left\{\left[\tau_{i}, \tau_{i}+\Delta_{i}\right]\right\}_{i=1}^{\infty} \subseteq[0, \infty)$ such that $\Delta_{i} \uparrow \infty$ as $i \uparrow \infty$, localization does not occur.

The next three theorems aim to improve on theorem 6 . The improvements are however only obtained at the expense of increasingly restrictive constraints on the coefficients $a$ and $b$ in equation (1.8). A constraint common to all three theorems is

$$
a^{\prime}(s) / s \in L^{1}(0, \delta) \quad \text { for some } \delta>0 .
$$

Supposing (6.11), one may define

$$
A(s)=\int_{0}^{s} a^{\prime}(r) / r \mathrm{~d} r
$$

for all $s \in[0, \infty)$.

Theorem 7. Suppose that (6.11) holds and

$$
[b(s)]_{+}=O(s A(s)) \quad \text { as } s \downarrow 0 .
$$

Then, if

$$
\lim _{i \uparrow \infty} \inf \left\{t A(\psi(t)): \tau_{i} \leq t \leq \tau_{i}+\Delta_{i}\right\}=\infty
$$

for a sequence of intervals $\left\{\left[\tau_{i}, \tau_{i}+\Delta_{i}\right]\right\}_{i=1}^{\infty} \subseteq[0, \infty)$ such that $\Delta_{i} \uparrow \infty$ sufficiently fast as $i \uparrow \infty$, localization does not occur. 
Proof. Recalling lemma 1, without loss of generality, we may suppose that $u_{0}(x) \leq 1$ for all $x \in[0, \infty)$ and $\psi(t) \leq 1$ for all $t \in[0, \infty)$. Furthermore, in the light of theorem 5 , we may assume that $\sigma_{0}=0$. Let $C>0$ be so large that

$$
[b(s)]_{+} \leq C s A(s) \quad \text { for all } s \in[0,1] .
$$

Suppose contrary to the assertion of the theorem, that there is an $X \in(0, \infty)$ such that

$$
\zeta(t) \leq X \quad \text { for all } t \in[0, \infty) .
$$

Let

$$
\kappa=2 X C^{-1} \exp (2 C X),
$$

and, utilizing (6.13), choose $i$ so large that

Define $\tilde{\psi}(t)$ by

$$
t A(\psi(t)) \geq \kappa \quad \text { for all } t \in\left[\tau_{i}, \tau_{i}+\Delta_{i}\right] .
$$

$$
t A(\tilde{\psi}(t))=\kappa \quad \text { for } t \in\left[\tau_{i}, \tau_{i}+\Delta_{i}\right]
$$

and note that plainly

$$
\psi(t) \geq \tilde{\psi}(t) \quad \text { for all } t \in\left[\tau_{j}, \tau_{i}+\Delta_{i}\right] .
$$

We assert that if $\Delta_{i}$ is large enough then we can find a $T \in\left[\tau_{i}, \tau_{i}+\Delta_{i}\right]$ and a $\sigma>0$ such that

$$
\begin{aligned}
& \sigma>\sup \{-b(s) / s: 0<s \leq \tilde{\psi}(T)\} \\
& \sigma\left(T-\tau_{i}\right)=\int_{0}^{\bar{\psi}(T)} a^{\prime}(s) /\{\sigma s+b(s)\} \mathrm{d} s
\end{aligned}
$$

and

$$
\sigma\left(T-\tau_{i}\right)>X .
$$

Assuming for the moment that this is true, define $z(x, t)$ in the closure of $D=(0, \infty) \times\left(\tau_{i}, T\right]$ by

$$
\int_{0}^{z(x, t)} a^{\prime}(s) /\{\sigma s+b(s)\} \mathrm{d} s=\left[\sigma\left(t-\tau_{i}\right)-x\right]_{+} .
$$

The function $z$ is a generalized solution of (1.8) in $D$. Moreover

$$
z(0, t) \leq z(0, T) \quad \text { for all } t \in\left[\tau_{i}, T\right],
$$

whilst by (6.21), (6.19), (6.16) and (6.17),

$$
z(0, T)=\tilde{\psi}(T) \leq \tilde{\psi}(t) \leq \psi(t) \quad \text { for all } t \in\left[\tau_{l}, T\right] .
$$

In addition,

Thus, by lemma 1 ,

$$
z\left(x, \tau_{i}\right)=0 \leq u\left(x, \tau_{i}\right) \quad \text { for all } x \in[0, \infty) .
$$

$$
z(x, t) \leq u(x, t) \quad \text { for all }(x, t) \in \bar{D} .
$$

However, this means that

$$
\zeta(T) \geq \sigma\left(T-\tau_{i}\right)
$$


which by (6.20) contradicts (6.15). The theorem is consequently proved by reductio ad absurdum, once we can show that if $\Delta_{i}$ is large enough there is a $T \in\left[\tau_{i}, \tau_{i}+\Delta_{i}\right]$ and $\sigma>0$ such that (6.18)-(6.20) hold.

For fixed arbitrary $T \in\left(\tau_{i}, \infty\right)$ consider (6.19) as an equation in $\sigma>\sigma^{*}$ where

$$
\sigma^{*}=\sup \{-b(s) / s: 0<s \leq \tilde{\psi}(T)\} \geq 0 .
$$

The left-hand side of (6.19) is an increasing function of $\sigma$ which tends to infinity as $\sigma \uparrow \infty$ and tends to $\sigma^{*}\left(T-\tau_{i}\right)$ as $\sigma \downarrow \sigma^{*}$. The right-hand side of (6.19) is a decreasing function of $\sigma$ which tends to zero as $\sigma \uparrow \infty$, and, since $\sigma_{0}=0$, tends to infinity as $\sigma \downarrow \sigma^{*}$ by lemma 10 . Thus, there must be a unique solution of (6.19), $\sigma$, such that (6.18) holds. Moreover, as a function of $T>\tau_{i}$, this solution is monotonic decreasing, and tends to zero as $T \uparrow \infty$. Consequently, it is possible to pick a pair $T \in(0, \infty)$ and $\sigma>0$ such that

$$
\sigma \tau_{i}<X
$$

and (6.18) and (6.19) hold. However, for this pair, substituting (6.14) in (6.19),

$$
\begin{aligned}
\sigma\left(T-\tau_{i}\right) & \geq \int_{0}^{\tilde{\psi}(T)} a^{\prime}(s) /\{\sigma s+C s A(s)\} \mathrm{d} s \\
& =\int_{0}^{\tilde{\psi}(T)} \frac{\mathrm{d}}{\mathrm{d} s}[\ln (\sigma+C A(s)) / C] \mathrm{d} s \\
& =\ln (1+C A(\tilde{\psi}(T)) / \sigma) / C \\
& =\ln (1+C \kappa /(\sigma T)) / C \\
& =\ln (1+2 X \exp (2 C X) /(\sigma T)) / C \\
& >\ln (2 X \exp (2 C X) /(\sigma T)) / C \\
& =2 X+\ln (2 X /(\sigma T)) / C .
\end{aligned}
$$

Thus

$$
\sigma T>2 X+\ln (2 X /(\sigma T)) / C .
$$

However this is only possible if $\sigma T>2 X$. Whence recalling (6.22), (6.20) holds. This confirms that if $\Delta_{i}$ is large enough we can choose a $T \in\left[\tau_{i}, \tau_{i}+\Delta_{i}\right]$ and a $\sigma>0$ such that (6.18)-(6.20) holds, and thereby completes the proof of the theorem.

THEOREM 8. Suppose that (6.11) holds and

$$
\left[b^{\prime}(s)\right]_{+}=O(A(s)) \quad \text { as } s \downarrow 0 .
$$

Let $\left\{\theta_{k}\right\}_{k=0}^{\infty},\left\{q_{k}\right\}_{k=0}^{\infty}$ and $\left\{p_{k}\right\}_{k=0}^{\infty}$ be defined inductively by (1.13)-(1.15). Then, if there exists a $k \geq 1$ and a $\delta>0$ such that

$$
\lim _{i \uparrow \infty} \inf \left\{p_{k}(t) q_{k}^{-\delta}(t) A(\psi(t)): \tau_{i} \leq t \leq \tau_{i}+\Delta_{i}\right\}>0
$$

for a sequence of intervals $\left\{\left[\tau_{i}, \tau_{i}+\Delta_{i}\right]\right\}_{i=1}^{\infty} \subseteq\left[\theta_{k}, \infty\right)$ such that $\Delta_{i} \uparrow \infty$ sufficiently fast as $i \uparrow \infty$, localization does not occur. 
Proof. We expand on an idea of Kersner [13]. Without loss of generality we may suppose that $u_{0}(x) \leq 1$ for all $x \in[0, \infty), \psi(t) \leq 1$ for all $t \in[0, \infty)$, and $\delta \leq 2$. Let $i$ and $\beta>0$ be such that

$$
A(\psi(t))>\beta p_{k}^{-1}(t) q_{k}^{\delta}(t) \quad \text { for all } t \in\left[\tau_{i}, \tau_{i}+\Delta_{i}\right] \text {. }
$$

Choose $C>0$ such that

$$
\left[b^{\prime}(s)\right]_{+} \leq C A(s) \quad \text { for all } s \in[0,1] .
$$

Now consider the function $z(x, t)$ defined by

$$
A(z(x, t))=p_{k}^{-1}(t) q_{k}^{\delta}(t)\left[\lambda-\left(x-x_{0}\right) C^{-1} q_{k}^{-\delta / 2}(t)\right]_{+}
$$

where $x_{0}=-\lambda C q_{k}^{\delta / 2}\left(\tau_{i}\right)$ for some real constant $\lambda$,

$$
0<\lambda<\beta \text {. }
$$

Defining the nonlinear differential operator $N(z)$ by

$$
N(z)=(a(z))_{x x}+(b(z))_{x}-z_{t}
$$

we find that

$$
\begin{aligned}
N(z)= & \left\{z / a^{\prime}(z)\right\}\left[p_{k}^{-2}(t) q_{k}^{\delta}(t)\left\{C^{-2}-\lambda \delta / 2\right\}\right. \\
& \left.+p_{k}^{-1}(t) A(z)\left\{\sum_{l=1}^{k}\left(\prod_{j=l}^{k} q_{j}(t)\right)+1-\delta / 2-C^{-1} q_{k}^{\delta / 2}(t) b^{\prime}(z) / A(z)\right\}\right]
\end{aligned}
$$

for $x<x_{0}+\lambda C q_{k}^{\delta / 2}(t)$ and $t \geq \tau_{i}$. Subsequently, by (6.25), there holds

$$
\begin{aligned}
N(z) \geq & \left\{z / a^{\prime}(z)\right\}\left[p_{k}^{-2}(t) q_{k}^{\delta}(t)\left\{C^{-2}-\lambda \delta / 2\right\}\right. \\
& \left.+p_{k}^{-1}(t) A(z)\left\{\sum_{i=1}^{k}\left(\prod_{j=1}^{k} q_{j}(t)\right)+1-\delta / 2-q_{k}^{\delta / 2}(t)\right\}\right]
\end{aligned}
$$

for all $x<x_{0}+\lambda C q^{\delta / 2}(t)$ and $t \geq \tau_{i}$. It follows that if we choose

$$
\lambda<2 \delta^{-1} C^{-2}
$$

then

$$
N(z)(x, t) \geq 0 \quad \text { for all } x \in\left[0, x_{0}+\lambda C q_{k}^{\delta / 2}(t)\right) \text { and } t \geq \tau_{i} .
$$

However, noting that

$$
N(z)(x, t)=0 \quad \text { for } x>x_{0}+\lambda C q_{k}^{\delta / 2}(t) \text { and } t \geq \tau_{i},
$$

one may deduce that $z$ is then a generalized subsolution of equation (1.8) in

$$
D=(0, \infty) \times\left(\tau_{i}, \tau_{i}+\Delta_{i}\right] .
$$

Moreover, utilizing (6.24), (6.26) and (6.27),

$$
u(x, t) \geq z(x, t) \quad \text { for all }(x, t) \in \bar{D} \backslash D .
$$

Hence, by the comparison principle of lemma 1 ,

$$
u(x, t) \geq z(x, t) \quad \text { for all }(x, t) \in \bar{D} .
$$


However, this implies

$$
\zeta(t) \geq x_{0}+\lambda C q_{k}^{\delta / 2}(t)=\lambda C\left\{q_{k}^{\delta / 2}(t)-q_{k}^{\delta / 2}\left(\tau_{i}\right)\right\} \quad \text { for all } t \in\left[\tau_{i}, \tau_{i}+\Delta_{i}\right] .
$$

It follows that if $\Delta_{i} \uparrow \infty$ as $i \uparrow \infty$ sufficiently fast that $q_{k}^{\delta / 2}\left(\tau_{i}+\Delta_{i}\right)-q_{k}^{\delta / 2}\left(\tau_{i}\right) \rightarrow \infty$, localization is excluded.

Corollary 8.1. Suppose that (6.11) and (6.23) hold and $\lim _{t \dagger \infty} \inf p_{k}(t) q_{k}^{-\delta}(t) A(\psi(t))>0$ for some $k \geq 1$ and $\delta>0$. Then there exists a $K>0$ and a $\tau \in[0, \infty)$ such that $\zeta(t) \geq K q_{k}^{\gamma}(t)$ for all $t \geq \tau$ where $\gamma=\min \{1, \delta / 2\}$.

THEOREM 9. Suppose that (6.11) holds and that

$$
a^{\prime}(s)=O(A(s)) \quad \text { and } \quad\left|b^{\prime}(s)\right|=O(A(s)) \quad \text { as } s \downarrow 0 .
$$

Then, irrespective of the function $\psi$, localization does not occur.

Proof. We use a comparison principle argument again. By hypothesis 3 , there is an $x_{0} \in(0, \infty)$, a $\delta>0$ and a $\rho \in\left(0, x_{0}\right)$ such that

$$
A\left(u_{0}(x)\right) \geq \delta \quad \text { for all } x \in\left[x_{0}-\rho, x_{0}+\rho\right] .
$$

Let $C>0$ be such that

$$
\left|b^{\prime}(s)\right| \leq C A(s) \quad \text { and } \quad a^{\prime}(s) \leq C A(s) \quad \text { for all } s \in(0, \delta] .
$$

Consider next the function $z(x, t)$ defined by

where

$$
A(z(x, t))=\lambda(t+1)^{-1}\left[\rho^{2}-\left(x-x_{0}\right)^{2}\right]_{+}
$$

$$
0<\lambda \leq \delta \rho^{-2} \text {. }
$$

With $N(z)$ given by $(6.28)$, one calculates that

$$
N(z)=(t+1)^{-1}\left\{z / a^{\prime}(z)\right\}\left\{A(z)-2 \lambda\left(x-x_{0}\right) b^{\prime}(z)-2 \lambda a^{\prime}(z)+4 \lambda^{2}\left(x-x_{0}\right)^{2}(t+1)^{-1}\right\}
$$

for $\left|x-x_{0}\right|<\rho$ and $t \geq 0$. Hence, dropping the last term in (6.34) and using (6.31),

$$
N(z) \geq(t+1)^{-1}\left\{z A(z) / a^{\prime}(z)\right\}\left\{1-2 \lambda C\left|x-x_{0}\right|-2 \lambda C\right\}
$$

for all $\left|x-x_{0}\right|<\rho$ and $t \geq 0$. Thus if $\lambda$ is chosen so small that

there holds

$$
1>2 \lambda C(1+\rho)
$$

Trivially, though

$$
N(z)(x, t) \geq 0 \quad \text { for all }\left|x-x_{0}\right|<\rho \text { and } t \geq 0 \text {. }
$$

$$
N(z)(x, t)=0 \quad \text { for all }\left|x-x_{0}\right|>\rho \text { and } t \geq 0 .
$$

So $z$ is a generalized subsolution of equation $(1.8)$ in $(0, \infty) \times(0, T]$ for any $T \in(0, \infty)$. Moreover, by (6.30), (6.32) and (6.33),

$$
\begin{array}{ll}
u_{0}(x) \geq z(x, 0) & \text { for all } x \in\left[x_{0}-\rho, x_{0}+\rho\right], \\
u_{0}(x) \geq 0=z(x, 0) & \text { for all } x \in\left[0, x_{0}-\rho\right) \cup\left(x_{0}+\rho, \infty\right),
\end{array}
$$


while

$$
\psi(t) \geq 0=z(0, t) \quad \text { for all } t \in[0, T] .
$$

Consequently, recalling lemma 1 ,

$$
u(x, t) \geq z(x, t) \quad \text { for all }(x, t) \in(0, \infty) \times(0, T] .
$$

Whence since $T>0$ was arbitrary,

$$
A\left(u\left(x_{0}, t\right)\right) \geq \lambda(t+1)^{-1} p^{2} \quad \text { for all } t \geq 0 .
$$

Armed with this conclusion though, we may invoke theorem 8 and lemma 1 once more to deduce that localization cannot occur. Actually, we can state the corollary below.

CoRollaRy 9.1 . Under the conditions of theorem 9 there is a $K>0$ such that $\zeta(t) \geq K|\ln t|^{1 / 2}$ for all $t \geq 1$.

COROLlaRy 9.2. Let $n \geq m$ in problem $\rho^{*}$. Then, irrespective of the function $\psi$, localization does not occur.

In the context of unsaturated soil-moisture flow, theorem 9 may be interpreted as indicating that for certain soils, soil-moisture penetration will occur without bound, even against the pull of gravity and even when a controlling boundary is desiccated.

We note that in theorems 7-9, the assumptions on the large-time behaviour of the boundarydata function $\psi$ are successively relaxed. However, since using elementary calculus it can be shown that (6.29) implies (6.23) whilst (6.23) implies (6.12), this relaxation clearly exacts an increasing price in the placing of restrictions on the coefficients $a$ and $b$ in (1.8). As an example of a pair of functions to which the basic hypotheses, hypotheses $1-4$, and (6.11), (6.12) apply, but not (6.23), we may take

$$
a(s)=s^{m} \quad \text { and } \quad b(s)=s^{n} \sin s^{-1}
$$

with

$$
m+1>n>m>1 \text {. }
$$

Whereas for an example of a pair which satisfy the basic hypotheses, (6.11), (6.12) and (6.23), but not (6.29), consider

$$
a(s)=\int_{0}^{s} r^{-1} \exp \left(-r^{-1}\right) \mathrm{d} r \quad \text { and } \quad b(s)=\int_{0}^{s} \exp \left(-r^{-1}\right) \mathrm{d} r .
$$

Theorems 7-9 are consistent with theorems 1 and 2. For if there exists a $v>0$ such that $b(s)>0$ for all $s \in(0, v]$ and $(6.11)$ and $(6.12)$ hold, we have

$$
\begin{aligned}
\int_{r}^{\nu} a^{\prime}(s) / b(s) \mathrm{d} s & \geq \int_{r}^{\nu} a^{\prime}(s) /[C s A(s)] \mathrm{d} s \\
& =\int_{r}^{\nu} \frac{\mathrm{d}}{\mathrm{d} s}[\ln (A(s)) / C] \mathrm{d} s \\
& =\ln (A(v) / A(r)) / C
\end{aligned}
$$


for some $C>0$ and all $r \in(0, v]$. So, letting $r \downarrow 0, a^{\prime}(s) / b(s) \notin L^{1}(0, v)$, and the set $S$ is necessarily empty.

The next theorem, which is the last in this section, indicates a further set of circumstances in which localization is excluded. In common with theorems 4 and 9 , this theorem indicates conditions on the coefficients $a$ and $b$ which infer that localization does not occur without any qualifications on the function $\psi$ in problem $\odot$.

THEOREM 10. Suppose that there is a $v \in(0, \infty)$ such that

$$
b(s) \leq 0 \quad \text { for all } s \in(0, v] .
$$

Then, irrespective of the function $\psi$, localization does not occur.

Proof. Without loss of generality we may suppose that $u_{0}(x) \leq v$ for all $x \in[0, \infty)$ and $\psi(t) \leq v$ for all $t \in[0, \infty)$. Furthermore, we may suppose that

$$
\lim _{t+\infty} \sup \psi(t)=0
$$

For $t \geq 0$, set

$$
M(t)=\sup \{u(x, t): x \in[0, \infty)\} .
$$

Since the constant $v$ is a generalized solution of equation (1.8) in all domains of the form (2.1), (2.2), there holds

$$
M(t) \leq v \quad \text { for all } t \in[0, \infty) .
$$

We distinguish between whether or not

$$
\lim _{t \uparrow \infty} \inf M(t)=0 .
$$

(i) Suppose that (6.35) holds. Then, by lemma 3,

$$
\begin{aligned}
\int_{0}^{\infty} x u_{0}(x) \mathrm{d} x & =\int_{0}^{\infty} x u(x, T) \mathrm{d} x-\int_{0}^{T} a(\psi(t)) \mathrm{d} t+\int_{0}^{T} \int_{0}^{\infty} b(u(x, t)) \mathrm{d} x \mathrm{~d} t \\
& \leq \int_{0}^{\infty} x u(x, T) \mathrm{d} x \\
& \leq M(T) \int_{0}^{\zeta(T)} x \mathrm{~d} x \\
& =\frac{1}{2} M(T) \zeta^{2}(T)
\end{aligned}
$$

for all $T>0$. Whence, in view of hypothesis $3, \lim _{T \dagger \infty} \sup \zeta(T)=\infty$.

(ii) Suppose on the other hand that (6.35) is not valid. In this case there is a $v \in(0, \infty)$ and a $\tau \in[0, \infty)$ such that

$$
M(t) \geq 2 v>v / 2 \geq \psi(t)
$$

for all $t \in[\tau, \infty)$. However, extending an argument in [8], we can subsequently show that given any $T \in(0, \infty)$ there exists a nonnegative function $\xi \in C([\tau, T])$ such that $u(\xi(t), t) \geq v$ for all $t \in[\tau, T]$. Whence, by lemma 11 , localization cannot occur in this case either. 
Corollary 10.1. Suppose that $\lambda \leq 0$ in problem $\rho^{*}$. Then, irrespective of the function $\psi$, localization does not occur.

Hydraulic conductivity is a nondecreasing function of moisture content in the theory of soil physics. Subsequently theorem 10 can be interpreted as stating that in soil-moisture infiltration in the direction of gravitational pull or in horizontal soil-moisture infiltration, soil-moisture penetration cannot be limited. Specifically, the particular boundary conditions imposed have no bearing on this conclusion.

\section{DISAPPEARANCE OF THE SUPPORT}

Consider problem $\rho^{*}$ with $\lambda>0$ and $m>n>0$. From theorem 2 we know that if $\lim _{t \uparrow \infty} \sup \psi(t)<\infty$, localization occurs, i.e.

$$
\lim _{t \uparrow \infty} \sup \zeta(t)<\infty .
$$

In the specific case $n<1$ though, Diaz and Kersner [6] have shown that if $\psi(t) \rightarrow 0$ in a suitable fashion as $t \rightarrow \infty$, then a much stronger result may be obtained, namely

$$
\lim _{t+\infty} \sup \zeta(t)=0
$$

That is to say, the support of the solution $u(x, t)$ disappears as $t \uparrow \infty$. On the other hand, if $\lim \inf \psi(t)>0$, then the converse $t+\infty$

is true.

$$
\lim _{t \uparrow \infty} \sup \zeta(t)>0
$$

The objective of this section is to extend the above-mentioned results of Diaz and Kersner. In particular we shall extend these results to problem $P$ in its general form.

The principal result on the disappearance of the support of the solution is the following.

THEOREM 11. Suppose that $\sigma_{0}<0$. Then, if $\lim _{t \uparrow \infty} \sup \psi(t)=0,(7.1)$ holds. Morcover, if there is a $T \in[0, \infty)$ such that

$$
\psi(t)=0 \quad \text { for all } t \geq T,
$$

there is a $T^{*} \in[T, \infty)$ such that

$$
\zeta(t)=0 \quad \text { for all } t \geq T^{*} .
$$

Proof. Pick $\sigma \in\left(\sigma_{0}, 0\right)$. Subsequently choose $v<\mu$ so small that $\sigma_{\nu}<\sigma$. By lemma 6 , there exists a $\tau \in[0, \infty)$ such that (4.3) and (4.4) hold. However, lemma 4 then tells us that

$$
\lim _{t \uparrow \infty} \sup \zeta(t) \leq I(\sigma, v)
$$

Whence, letting $v \downarrow 0,(7.1)$ follows. If now (7.3) holds, lemma 4 tells us immediately that (7.4) is the case.

In a sense the assumptions $\sigma_{0}<0$ and $\lim _{t \uparrow \infty} \sup \psi(t)=0$ in theorem 11 are essential. This can be seen by considering the following theorems. 
THEOREM 12. Suppose that $\sigma_{0}=0$ and there are real numbers $\rho$ and $\delta>0$ such that (6.1) and (6.2) hold. Then, (7.2) holds.

THEOREM 13. Suppose that $\sigma_{0}=0$. Then, if there is a $\tau \in[0, \infty)$ such that $\psi(t)>0$ for all $t \in[\tau, \infty),(7.2)$ holds.

THEOREM 14. If

$$
\lim _{i \rightarrow \infty} \inf \left\{\psi(t): \tau_{i} \leq t \leq \tau_{i}+\Delta_{i}\right\}>0
$$

for a sequence of intervals $\left\{\left[\tau_{i}, \tau_{i}+\Delta_{i}\right]\right\}_{i=1}^{\infty} \subseteq[0, \infty)$ such that $\tau_{i} \uparrow \infty$ and $\Delta_{i} \nrightarrow 0$ as $i \uparrow \infty,(7.2)$ holds.

Theorem 15. Suppose that $\sigma_{0}>0$. Then, if inf $\left\{\psi(t): \tau_{i} \leq t \leq \tau_{i}+\Delta_{i}\right\}>0$ for a sequence of intervals $\left\{\left[\tau_{i}, \tau_{i}+\Delta_{i}\right]\right\}_{i=1}^{\infty} \subseteq[0, \infty)$ such that $\tau_{i} \uparrow \infty$ and $\Delta_{i} \nrightarrow 0$ as $i \uparrow \infty,(7.2)$ holds.

THEOREM 16. Suppose that (6.11) and (6.12) hold. Then, if

$$
\lim _{i \neq \infty} \inf \left\{t A(\psi(t)): \tau_{i} \leq t \leq \tau_{i}+\Delta_{i}\right\}>0
$$

for a sequence of intervals $\left\{\left[\tau_{i}, \tau_{i}+\Delta_{i}\right]\right]_{i=1}^{\infty} \subseteq[0, \infty)$ such that $\Delta_{i} \uparrow \infty$ sufficiently fast as $i \uparrow \infty$, (7.2) holds.

Theorems 12,13 and 14 follow from lemmas 9,8 and 7 respectively. Theorems 15 and 16 may be obtained by adapting the proofs of theorems 5 and 7 respectively. The details of the proofs may be easily constructed.

Note that theorem 13 is not covered by theorem 12 . The functions given by $(6.7)$ and $(6.8)$ satisfy the hypotheses of theorem 13 but not those of theorem 12 .

Note too that the conditions of theorem 14 do not exclude the possibility that lim inf $\psi(t)=0$.

As corollaries to theorems 11,12 and 14 we have the following results.

Corollary 11.1. Let $\lambda>0$ and $n \leq 1$ in problem $P^{*}$. Then if $\lim _{t \dagger \infty} \sup \psi(t)=0,(7.1)$ holds. Moreover, if there is a $T \in[0, \infty)$ such that (7.3) holds, there is a $T^{*} \in[T, \infty)$ such that $(7.4)$ holds.

Corollary 12.1. Let $\lambda>0$ and $n>1$ in problem $\rho^{*}$. Then, (7.2) holds.

Corollary 14.1. Let $\lambda>0$ and $n \leq 1$ in problem $\odot^{*}$. Then, if (7.5) holds for a sequence of intervals $\left\{\left[\tau_{i}, \tau_{i}+\Delta_{i}\right]\right\}_{i=1}^{\infty} \subseteq[0, \infty)$ such that $\tau_{i} \uparrow \infty$ and $\Delta_{i} \leftrightarrow 0$ as $i \uparrow \infty,(7.2)$ holds.

Thus for problem $\rho^{*}$ the only case in which the disappearance of the support of the solution is unresolved is that for $\lambda>0, n \leq 1$ when $\psi(t)$ oscillates as $t \uparrow \infty$ so that

$$
\lim _{t \uparrow \infty} \inf \psi(t)=0<\lim _{t \uparrow \infty} \sup \psi(t)
$$

with increasingly sharper peaks. 
Acknowledgements-With alternative proofs, corollaries 2.1 and 9.2 and weaker forms of corollary 3.1 and theorem 10 of this paper were presented at the meeting "Nonlinear parabolic equations: qualitative properties of solutions" held at the Second University of Rome in April 1985. The author would like to apologise to the organizers of this meeting, L. Boccardo and A. Tesei, and also to M. Mimura and T. Nagai whom refer to these results in the proceedings of the meeting, for his failure to meet the deadline for their inclusion in the proceedings.

The author is grateful to $\mathrm{R}$. Kersner for permission to quote and profit from unpublished results contained in [13].

\section{REFERENCES}

1. Aronson D. G., The porous medium equation, in Nonlinear Diffusion Problems (Edited by A. Fasano and M. Primicerio), Lecture Notes in Mathematics 1224, 1-46. Springer, Berlin (1986).

2. Barenblatt G. I. \& Zel'dovich Ya. B., On the dipole-type solution in problems of unsteady gas filtration in the polytropic regime, Prikl. Mat. Mekh. 21, 718-720 (1957). (In Russian.)

3. Bear J., Zaslavsky D. \& Irmay S., Physical Principles of Water Percolation and Seepage. United Nations Educational Scientific and Cultural Organization, Paris (1968).

4. Diaz J. I. \& Kersner R., Non existence d'une des frontières libres dans une équation dégénérée en théorie de la filtration, C.R. Acad. Sci. Paris Sér. I Math. 296, 505-508 (1983).

5. Diaz J. I. \& KeRSNER R., On a nonlinear degenerate parabolic equation in infiltration or evaporation through a porous medium, J. diff. Eqns 69, 368-403 (1987).

6. Diaz J. I. \& Kersner R., On the behaviour and cases of nonexistence of the free boundary in a semibounded porous medium, J. math. Analysis Applic. 132, 281-289 (1988).

7. Guding B. H., Properties of solutions of an equation in the theory of infiltration, Archs ration. Mech. Analysis 65, 203-225 (1977).

8. Gilding B. H., The occurrence of interfaces in nonlinear diffusion-advection processes, Archs ration. Mech. Analysis 100, 243-263 (1988).

9. Gilding B. H., Improved theory for a nonlinear degenerate parabolic equation, Annali Scu. norm. sup. Pisa Cl. Sci. (4) (to appear). (Previously appearing in provisional form as: Twente University of Technology Department of Applied Mathematics Memorandum 587, (1986).)

10. Kalashnikov A. S., The nature of the propagation of perturbations in processes that can be described by quasilinear degenerate parabolic equations, Trudy Sem. Petrovsk. 1, 135-144 (1975). (In Russian.)

11. Kalashnikov A. S., Some problems of the qualitative theory of non-linear degenerate second-order parabolic equations, Russ, math. Survs 42, 169-222 (1987), translation of Usp. mat. Nauk 42, 135-176 (1987).

12. KERSNER R., Localization conditions for thermal perturbations in a semibounded moving medium with absorption, Moscow Univ. Math. Bull. 31, 90-95 (1976), translation of: Vest. Moskov. Univ. Ser. I Mat. Mekh. 31, 52-58 (1976).

13. Kersner R., Private communication (1986).

14. Matano H., Nonincrease of the lap-number of a solution for a one-dimensional semilinear parabolic equation, J. Fac. Sci. Univ. Tokyo Sect. IA Math. 29, 401-441 (1982).

15. Peletier L. A., The porous media equation, in Applications of Nonlinear Analysis in the Physical Sciences (Edited by H. Amann, N. Bazley and K. Kirchgässner), pp. 229-241. Pitman, Boston (1981).

16. Swartzendruber D., The flow of water in unsaturated soils, in Flow through Porous Media (Edited by R. J. M. DE WIEST), pp. 215-292. Academic Press, New York (1969). 